\author{
Gdańsk 2019, Nr. 41 \\ https://doi.org/10.26881/sgg.2019.41.22 \\ Katarzyna Taborska \\ Akademia im. Jakuba z Paradyża \\ (Gorzów Wielkopolski) \\ https://orcid.org/0000-0002-0038-9488

\section{Zur narrativ-bildenden Funktion von Ephemera im ,Grenzgebiet des Augenblicks‘}

Das Ziel des Beitrags ist die Darstellung der narrativ-bildenden Funktion von ausgewählten ephemeren Texten aus dem Jahre 1945, in dem das brandenburgische Landsberg an der Warthe eine plötzliche Metamorphose zum polnischen Gorzów Wielkopolski erfuhr. Das Schicksal dieser Stadt erscheint repräsentativ für die Geschichte von Orten, die nach dem Zweiten Weltkrieg infolge der Grenzverschiebungen ihre Identität verändert haben. Im Beitrag werden sogenannte Prä-Narrative dargestellt, d.h. Erzählbausteine, die sich in ephemeren Texten aus dem, Grenzgebiet des Augenblicks' entdecken lassen und die mit der Kreierung eines kollektiven Bildes des jeweiligen Ortes zusammenhängen. Diese Prä-Narrative sind ein Beitrag zur Geschichte der Beziehungen zwischen Menschen aus drei Kulturen: der deutschen, der polnischen und der russischen. Im Aufsatz werden die Fallstudien und die Metasprache aus der Monographie von Katarzyna Taborska Literatura miejsca. Piśmiennictwo postlandsberskie [,Literatur des Ortes. Das post-landsbergische Schrifttum'], Gorzów Wielkopolski 2017, aufgegriffen.

Schlüsselwörter: ephemere Drucke, Grenzgebiet des Augenblicks, Prä-Narrative des neuen Grenzgebiets, deutsch-russische Beziehungen, deutsch-polnische Beziehungen

Ephemera as pre-narratives. Case analysis of the 'borderland of the moment'. - The aim of the article is to show the 'narrative' role of selected ephemeral texts created in 1945 at the time of the sudden metamorphosis of the Brandenburg city of Landsberg an der Warthe into Polish-speaking Gorzów Wielkopolski. The situation of this city can be treated as an exemplification of the history of places which, after the Second World War, changed their identity as a result of shifting borders. The article presents the origins of narration (pre-narratives) related to the creation of ideas about the place that can be discovered in the transitory writings of the 'borderland of the moment'. They are a contribution to the history of the relationships of people from the cultures of three languages: German, Polish and Russian. The elaboration uses the analysis and meta-language of Katarzyna Taborska's monograph Literatura miejsca. Piśmiennictwo postlandsberskie ['Literature of place. Postlandsberg writings'], Gorzów Wielkopolski 2017.

Keywords: ephemera, borderland of the moment, pre-narrations of the new borderland, German-Russian relations, German-Polish relations 


\section{Inspirationen und terminologische Vorabklärung}

Gegenstand des vorliegenden Beitrags ist die narrativ-bildende Funktion von ausgewählten ephemeren Texten, die 1945 im neuen deutsch-polnischen Grenzgebiet entstanden. Für die Zwecke dieses Beitrags lässt sich diese ,narrativ-bildende Funktion' folgendermaßen verstehen:

- Erstens: Die ausgewählten Texte können als Erzählungen aufgefasst werden, die bestimmte Vorstellungen über die dynamische Entwicklung der Realität am Ende des Zweiten Weltkriegs mit angestoßen haben;

- zweitens: Die Ephemera erscheinen als Prä-Narrative, d.h. als erste Erzählbausteine für spätere Gründungsgeschichten aus dem betreffenden Gebiet;

- drittens: die Ephemera lassen sich als potentieller Forschungsgegenstand der Literaturwissenschaft auffassen, der jedoch nicht zu deren standardmäßigem Interessenbereich gehört.

Der Begriff, neue deutsch-polnische Grenzgebiete' ist einer der Fachtermini, die ich für die Zwecke meiner Studie Literatura miejsca. Piśmiennictwo postlandsberskie (TABORSKa 2017, dt.: ,Literatur des Ortes. Das post-landsbergische Schriftum) geprägt habe. In dieser Monographie werden aus literaturanthropologischer Perspektive Texte erörtert, die während der einschneidenden Transformation des Ortes „Landsberg an der Warthe“ in „Gorzów Wielkopolski“ entstanden. Die Hauptstadt der historischen Landschaft Neumark erscheint in der Studie als repräsentatives Beispiel für deutschsprachige Orte, die infolge von Grenzverschiebungen und großen Migrationen ganzer Bevölkerungsgruppen - als Folge der Beschlüsse der Konferenzen von Jalta und Potsdam - ihre Identität verändert haben.

Der vorliegende Beitrag geht auf die Fallstudien aus der Monographie Literatura miejsca zurück, die an die Thematik des Artikels angepasst, umgearbeitet und ins Deutsche übersetzt wurden. Er enthält aber auch Inhalte, die in das Buch nicht aufgenommen wurden und die sich daher als dessen deutschsprachige Ergänzung lesen lassen. Im Beitrag wird der Versuch unternommen, Elemente des in der Monographie vorgeschlagenen analytischen Modells in deutscher Sprache zu präsentieren.

Der Begriff, neue deutsch-polnische Grenzgebiete bedarf einer Präzisierung. ${ }^{1}$ Das Adjektiv ,neu' verweist auf den gesellschaftlichen, politischen, kulturellen und ethnischen Wandel, der sich in den 1945 an Polen angeschlossenen Gebieten vollzog und immer noch vollzieht. Das Wort lenkt die Aufmerksamkeit auf die veränderten territorialen, demographischen sowie ethnisch-nationalen Verhältnisse nach dem Zweiten Weltkrieg. Die Pluralform ,Grenzgebiete betont die Uneinheitlichkeit des Territoriums, das in den polnischen Staat eingegliedert wurde. Unter den ,neuen Grenzgebieten' gibt es Orte, in die das polnische Element als Bestandteil der kulturellen Identität erst 1945, im Zuge der Westverschiebung Polens, vordrang (vgl. ТАвORSKa 2017: 15). Zu ihnen gehört eine der beiden Hauptstädte der heutigen Woiwodschaft Lebus (polnisch: województwo lubuskie): Gorzów Wielkopolski, das frühere brandenburgische Landsberg an der Warthe. Die erste Entstehungsphase des neuen Grenzgebiets, das sich um diese Stadt herum bildete, ist eine Zeit vielfältiger, gravierender, oft vehementer Veränderungen. Diese Etappe in der Geschichte des neuen Grenzgebiets

1 Natürlich ließe sich über den Gehalt und den Bedeutungsumfang des Terminus ,Grenzgebiet' diskutieren, dies ginge jedoch über den Rahmen dieses Beitrags hinaus. 
bezeichne ich als, Grenzgebiet des Augenblicks' (vgl. TABORSKa 2017: 16); es entsteht in der zweiten Hälfte der 1940er Jahre, als der Ort eine plötzliche und radikale Änderung seiner Identität erfährt. Zeugnis von der Existenz des, Grenzgebiets des Augenblicks' legen u.a. Kulturtexte aus der damaligen Zeit ab.

Im Teil II des Beitrags (Fallstudien aus dem, Grenzgebiet des Augenblicks') wird der narrativ-bildende Charakter folgender ephemerer Schriftstücke veranschaulicht:

- ein russischsprachiger Propagandaspruch, der an der deutsch-polnischen Grenze verbreitet wurde (Abs. 1),

- zwei deutschsprachige Aufrufe (Abs. 1 und 3),

- ein deutschsprachiger Artikel aus der sowjetischen ephemeren Zeitschrift „Front Illustrierte“, die zu Propagandazwecken gedruckt wurde (Abs. 2).

Dargestellt wird im Folgenden auch die Geschichte eines nicht erhaltenen deutsch-polnischen Plakats, dessen Existenz heute nur noch in Berichten von Zeitzeugen belegt ist (Abs. 2).

Aus Platzgründen mussten zahlreiche polnische Ephemera ausgeklammert werden. Immerhin ist deren Anzahl so beträchtlich, dass sie es dem polnischen Historiker Dariusz Aleksander Rymar erlaubte, eine Sammlung von Quellentexten zu Landsberg bzw. Gorzów Wielkopolski aus den Jahren 1945-1948 herauszugeben (vgl. RymaR (Hg.) 2001). Der Herausgeber hat das im Staatsarchiv in Gorzów Wielkopolski vorhandene Material dahingehend ausgewertet, dass er daraus bewusst einen historiographischen Text gestaltete. Diese Absicht lässt sich sowohl an der Struktur der Monographie als auch an der vielsagenden Überschrift des Inhaltsverzeichnisses erkennen, die lautet: „Inhalt der ,Chronik der Stadt Gorzów““ (vgl. Rymar (Hg.) 2001: 13). Diese aus der Feder eines Historikers stammende Publikation führt den offensichtlich narrativ-bildenden Charakter ephemerer Drucke vor Augen - prä-narrativer Texte, die im Keim eine Geschichte enthalten und den Erzählungen über Orte im ,neuen Grenzgebiet' zugrunde liegen.

\section{Fallstudien aus dem, Grenzgebiet des Augenblicks}

\section{1.}

Вот она, проклятая Германия!“ - ,DAS IST Es, das verfluchte DeUtsChLAND!', so lautet in wörtlicher Übersetzung die emotionsgeladene elliptische Aussage auf einem der Schilder, die Sowjet-Soldaten an mehreren Stellen der ehemaligen deutsch-polnischen Grenze aus der Vorkriegszeit anbrachten (Abb. 1).

Dieser Spruch lässt verschiedene Funktionen erkennen. Er gibt Auskunft über den Grenzübertritt zwischen Polen und Deutschland (informative Funktion), bringt Emotionen zum Ausdruck, die ein bestimmter Ort bzw. ein Staatsgebiet hervorruft (expressive Funktion) und löst revanchistische Verhaltensweisen sowie Machtansprüche aus (impressive Funktion). Die Expressivität der Aussage verdeutlicht und kreiert zugleich die Empfindungen sowjetischer Soldaten und ihr Verhältnis zu diesem Ort (vgl. TABORSKA 2017: 57).

An dieser knappen, zweiteiligen Aussage fällt eine formale Raffiniertheit auf. Der erste Teil (,Das ist es ${ }^{c}$ ) leitet zur Präsentation des Ortes über. Die Ausdruckskraft des Epithetons, verflucht' wird durch das Ausrufezeichen am Satzende verstärkt. Der Text fügt sich in die Poetik gängiger politischer Propaganda-Aussagen voller Vereinfachungen und Verallgemeinerungen 


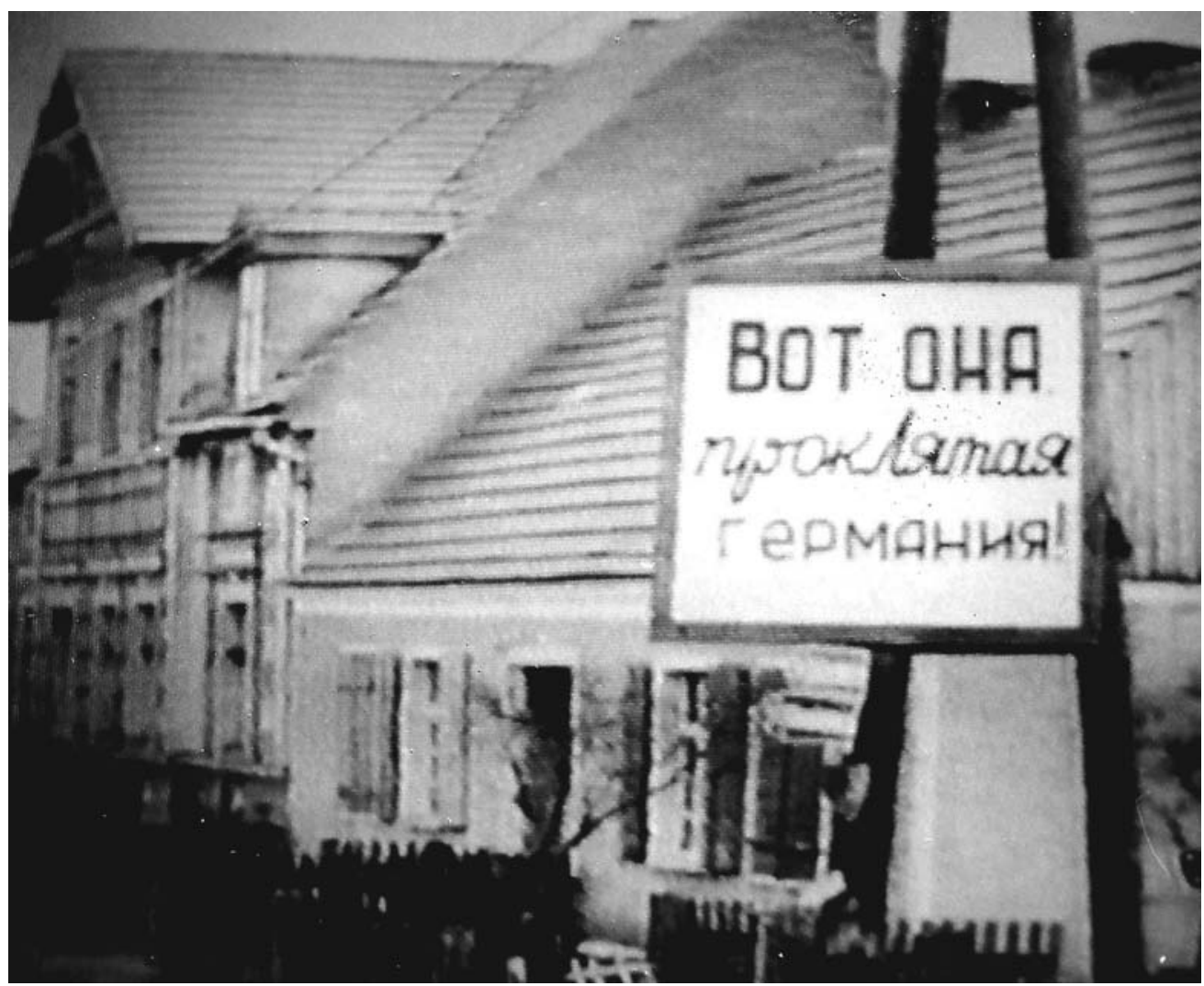

Abb. 1. Quelle: Adamczewski (2012: 11)

ein, hebt sich von ihnen aber durch seine Expressivität ab. Aus gattungspoetischer Perspektive erscheint der angeführte Spruch als ein kunstvoll gestaltetes propagandistisches Epigramm mit drei Aufgaben. Erstens: Er soll betonen, dass der Rezipient das Territorium des Feindes betritt. Zweitens: Der Text soll negative Einstellungen gegenüber den Deutschen verstärken. Drittens: Er soll Reaktionen hervorrufen und Verhaltensweisen provozieren, die typisch für die jeweiligen Sieger sind (vgl. TAвORSKa 2017: 57-58).

Der russische Slogan tritt in unterschiedlichen Erzählformen auf, u.a. in Berichten von Zeitzeugen. So findet sich der Spruch „Вот она, проклятая Германия!“ im Schaffen Elena Rzhevskaias (Pseudonym von Elena Kagan), einer Dolmetscherin des militärischen Nachrichtendienstes der Sowjetunion, des sogenannten SMERSch. Er wird in einem von mehreren ,Mikro-Romanen' zitiert, aus denen die Autorin ihren Kriegsbericht zusammenbaut. ${ }^{2}$ Die polnische Übersetzung dieses Erzähltextes von Rzhevskaia, unter dem Titel Berlin, maj 1945 [Berlin, Mai 1945], erschien drei Jahrzehnte nach dem Krieg (Ṙ̇Ewska 1975). Im Kapitel Droga do Berlina [,Der Weg nach Berlin'], das die einzelnen Ortschaften auf dem Weg der

2 Das russische Original ist unter der folgenden Adresse zu finden: http://militera.lib.ru/memo/russian/ pzhevskaya_em2/index.html (27.05.2019). 
Roten Armee beschreibt, wird das angeführte Epigramm Bestandteil der Erzählung über die Sieger. Der polnischen Übersetzung von Aleksander Bogdański ist zu entnehmen, dass einer der Rotarmisten die Parole vom „verfluchten Deutschland“ aus eigener Initiative mit Holzteer auf eine Tafel angebracht hat. Um diese Aufschrift herum entwickelt Rzhevskaia ihre Erzählung vom Einmarsch der sowjetischen Truppen in Deutschland. Dabei entwickelt sie ihre eigene Vorstellung vom Urheber dieses Textes: einem Soldaten der Roten Armee, der über vier Jahre mit dem deutschen Feind gekämpft hatte, bis er endlich in dessen Territorium gelangte. Rzhevskaia will die Verantwortung für den Inhalt des Epigramms nicht den sowjetischen Propagandisten zuschreiben, sie verleiht ihm vielmehr einen individuellen Charakter, indem sie ,irgendeinen Soldaten“ zu seinem Autor macht. Infolge dessen bekräftigt sie den emotionalen und scheinbar magischen Gehalt des an der deutsch-polnischen Grenze angebrachten Textes. Sie liefert eine Rechtfertigung für die Generalisierungen, die der Spruch vermittelt: Es ist ein Einzelner, ein rachsüchtiger Soldat, der das Prinzip der kollektiven Verantwortung anwendet; dieses Prinzip ergibt sich hier nicht aus dem staatlichen propagandistisch-erzieherischen Programm. Last but not least hält Rzhevskaia also die Möglichkeit offen, das Epigramm jenseits einer Propaganda-Dimension zu lesen (vgl. Taвorska 2017: 59-60).

AdamCZewski (2012), der die Erzählstrategie von Rzhevskaia im Vorwort zu seiner Sammlung historischer Reportagen kommentiert, hält Folgendes fest:

„Für mich unterliegt es keinem Zweifel, dass die russische Aufschrift ,Wot ona, proklataja Germanija! an der Grenze zum Dritten Reich nicht auf die eigene Initiative der Soldaten zurückging. Es war eine gezielte Aktion des politischen Apparats der Roten Armee, die den Soldaten bewusst machen sollte, dass sie das Territorium des Feindes betreten und dass sie hier - so legt der Spruch nahe - vergewaltigen, plündern, töten und alles niederbrennen dürfen..." (ADAMCZEwSKI 2012: 9-10) ${ }^{3}$

Vor diesem Hintergrund erscheint das besagte Epigramm als Pendant zu deutschfeindlichen Texten, die Stalins Propaganda hervorgebracht hat. Wie intensiv die Erinnerung an den Stil der damaligen sowjetischen Rhetorik ist, davon zeugen die Memoiren des Landsbergers Hartmut Meyer-Ort (2008), der das tragische Schicksal seiner Heimatstadt nach dem Einmarsch der Roten Armee am 30. Januar 1945 miterlebt hatte. Die Stadt wurde ohne Gegenwehr besetzt. Als die Sowjet-Soldaten in die Stadt einrückten, gab es dort keine deutschen Truppen mehr. Trotzdem wurde die Innenstadt niedergebrannt und zerstört; Raub und Vergewaltigung waren an der Tagesordnung. Viele Landsberger begingen Selbstmord. Im Folgenden gehe ich auf die Aufzeichnungen Meyer-Orts ein, deren wesentlicher Kompositionsbestandteil ein Kampfappell an die Rotarmisten ist - ein Aufruf, den man als eine Art Erweiterung des propagandistischen Epigramms über das „verfluchte Deutschland“ lesen kann. Wohlgemerkt fehlt in Meyer-Orts Text sowohl die Quelle des Zitats als auch eine Angabe dazu, wer den Aufruf ins Deutsche übersetzt hat. Es gibt auch sonst keinerlei Anmerkungen.

An dem angeführten Aufruf fällt der präzise Rahmenaufbau auf. Genauso wie im Fall des Epigramms besteht das Wesen des Appells darin, das Bedürfnis nach Rache und Vergeltung zu wecken und jegliche Spur von Empathie zu unterdrücken:

3 Alle Übersetzungen der Zitate aus der polnischsprachigen Sekundärliteratur stammen von der Übersetzerin dieses Beitrags. 
„Die Russen marschierten in Landsberg ein mit Ilja Ehrenburgs Aufruf im Tornister: ,Tötet, ihr tapferen Rotarmisten, tötet! Es gibt nichts an den Deutschen, was unschuldig ist. Folgt den Anweisungen des Genossen Stalin und zerstampft das faschistische Tier in seiner Höhle. Brecht mit Gewalt den Rassenhochmut der germanischen Frauen, nehmt sie als rechtmäßige Beute. Tötet, ihr tapferen vorwärtsstürmenden Rotarmisten, tötet!'

Das Schreien der gejagten und vergewaltigten Frauen konnte man, besonders des Nachts, [...] hören [...]. Helfen konnte niemand. Ausgangssperre, keine Straßenbeleuchtung. Man sah aus dem Fenster das eine oder andere brennende Haus den Himmel erhellend. Jeder hoffte, hoffentlich geht der Kelch [...] an diesem Haus vorbei." (MEYER-ORT 2008: 178)

Übrigens würde Adamczewskis Interpretation des propagandistischen Epigramms bei Tomas Venclova, der die Ilja-Ehrenburg-Biographie von Ewa ZARZYCKA-BÉRARD (2002) kommentiert hat, wahrscheinlich keine Anerkennung finden. Venclova hätte vermutlich auch die Urheberschaft des von Meyer-Ort zitierten Appells an die Rotarmisten bestritten. ${ }^{4}$ Meine Vermutungen stütze ich auf folgende Passagen aus Venclovas Rezension:

„Während des spanischen Bürgerkriegs hat sich Ehrenburg als Journalist hervorgetan, und während des Zweiten Weltkriegs ist er zum berühmtesten Journalisten weltweit geworden. Ohne Zweifel hat er zum Sieg über Hitler beigetragen. Dennoch will die Biographin Ewa Zarzycka-Bérard die dunklen Seiten seiner journalistischen Leistung nicht verschweigen. Ehrenburg ahmte bestimmte Vorbilder der französischen Rhetorik nach, die nicht gerade die besten waren. Er brüstete sich damit, den Spitznamen ,Fritz' (der ungefähr das gleiche wie das französische ,boche' bedeutet) erfunden zu haben. Er propagierte Hass auf die Deutschen an sich und behauptete, sie seien keine Menschenwesen, sondern gemeine, niederträchtige Monstren. Von ihm stammen folgende Worte: ,Wir zeigen ihnen, was der Krieg bedeutet. Wir werden ihnen die Zähne ausschlagen. Wir werden ihnen die Fingernägel ausreißen. Wir werden den Deutschen zeigen, was das Feuer der Gerechtigkeit ist: Mit diesen Worten hat Ehrenburg nicht nur zu den wilden Exzessen der sowjetischen Truppen auf deutschen Gebieten beigetragen, sondern er hat Goebbels dessen Arbeit wesentlich erleichtert. Antisemiten wiederholen bis heute die in der nationalsozialistischen Presse kolportierte Information, Ehrenburg habe zur Vergewaltigung jeder deutschen Frau aufgerufen. So war es aber nicht. Im Gegenteil: Wiktor Abakumow, Leiter der sowjetischen Gegenspionage, berichtete im März 1945 an Stalin, dass Ehrenburg auf Versammlungen gegen Schändungen und Plünderungen auftritt. Am 14. April 1945 erschien in der Tageszeitung „Prawda“ der berühmte Aufsatz Genosse Ehrenburg vereinfacht, in dem man betonte, dass es gute und schlechte Deutsche gibt; man kann sich des Eindrucks nicht erwehren, dass Stalin mit der für ihn typischen Perfidie Abakumows Denunziation ausnutzte, indem er Ehrenburg wegen dessen Undiszipliniertheit ermahnte und gleichzeitig die Schuld an den Verbrechen auf ihn abwälzte.“ (VenClOva 2003)

Das dargestellte Material veranschaulicht, dass ephemere Drucke nicht nur eine Quelle vielfältiger Erzählungen sind, die Vorstellungen von der Realität kreieren. Auf Ephemera gehen durchaus auch gegenwärtige Streitfragen zurück - Kontroversen um die Interpretation und sogar die Urheberschaft dieser Texte. Wohlgemerkt beginnt Ewa Zarzycka-Bérard das Kapitel, in dem sie Ehrenburgs Schaffen während des Zweiten Weltkriegs bespricht, mit einem Motto von Joseph Goebbels, wonach die Russen keine

4 Die Frage der umstrittenen - und bis heute nicht belegten - Autorschaft Ilja Ehrenburgs an dem angeführten Schändungsaufruf wird ausführlich diskutiert in: [Anonym] „Der Spiegel“ 36/1962 (05.09.1962), S. 71-76 (https://www.spiegel.de/spiegel/print/d-45141455.html, 15.06.2019). 
Menschen, sondern Vieh seien (vgl. ZarzyCKa-BÉrard 2002: 190). Somit zeigt sie die Zusammenhänge zwischen Propagandaschriften des Dritten Reiches und denjenigen der Sowjetunion auf.

2.

Gleichzeitig mit dem Aufsatz Genosse Ehrenburgvereinfacht erschien eine zu Propagandazwecken aufbereitete Ausgabe der ephemeren Zeitschrift „Front Illustrierte", die sich an deutsche Frontsoldaten richtete (Abb. 2). Ähnlich wie in der Tageszeitung „Prawda“ hielt man sich auch in diesem deutschsprachigen ephemeren Druck im Hinblick auf seine intendierten Rezipienten damit zurück, ein eindeutig negatives Bild der deutschen Gesellschaft und des Deutschtums zu vermitteln.

Bei diesem knappen Bildbericht handelt es sich um einen Text, der die von Propaganda-Maßnahmen gestaltete Medienlandschaft in der ersten Entstehungsphase des ,neuen Grenzgebiets' dokumentiert. Der Text wurde 2005 wieder in Erinnerung gerufen, zusammen mit dem Aufruf des letzten deutschen Bürgermeisters von Landsberg, Paul Schultz, der von der sowjetischen Besatzungsmacht eingesetzt worden war. Die beiden deutschsprachigen Schriftstücke wurden, neben anderen gattungsmäßig sehr unterschiedlichen Texten ehemaliger Landsberger, in einen von HASSE-Dresing (Hg.) (2005) herausgegebenen Sammelband aufgenommen. Der Band schildert die Verhältnisse in der Stadt nach dem Einmarsch der Rotarmisten. Der Bildbericht und der Appell von Paul Schultz tragen hier die gemeinsame Überschrift Zwei Dokumente der „anderen Seite“. Ein solcher Titel weist darauf hin, dass sich die Herausgeber von den dargestellten Zeitzeugnissen distanzieren, zugleich aber ihren historischen Wert hoch schätzen.

Der präsentierte Bildbericht hat einen propagandistischen, „magischen“ Titel, der die Existenz einer friedlichen Welt unter der Besatzungsmacht suggeriert. Die Überschrift „In den von Sowjettruppen besetzten deutschen Städten herrscht Ruhe und Ordnung " übernimmt zugleich die Funktion eines Leadsatzes, der die Kernaussage auf den Punkt bringt. Das Text-Bild-Gefüge veranschaulicht die Lage der Deutschen nach dem Einmarsch der Rotarmisten. Begleitet wird die Erzählung durch geradezu idyllische Fotos von Deutschen und Russen, die in Landsberg zusammenkommen.

Der Text besteht aus zwei Teilen: einem allgemeinen und einem, der sich speziell auf die Landsberger Verhältnisse bezieht. Er beginnt mit der Anklage gegen die Nazipropaganda, d.h. „Hitler und seine Spießgesellen“. Diese werden beschuldigt, die Lüge zu verbreiten, dass die Rote Armee „die friedliche Bevölkerung in den von ihr besetzten Städten“"vernichte. Weiter verkündet der Text, dessen Autor seine Identität nicht verrät, Folgendes:

„Diese Lügenmärchen über, sowjetische Greueltaten ' haben den Zweck, die Soldaten zur Fortsetzung des sinnlosen Widerstands anzustacheln. Durch ihre Lügen versetzen die Nazis die friedliche Bevölkerung in Panik und stürzen die deutschen Frauen und Kinder, die aus den Städten fliehen, ins Verderben."

Der zweite Absatz der Einführung steht im Widerspruch zum russischen prä-narrativen Schlagwort ,Wot ona, proklataja Germanija!' und ist sicherlich nicht dessen Fortsetzung. In dieser Passage fallen die - leicht abgewandelte - Wiederholung der Überschrift und ein mit Ausrufezeichen beendeter Satz auf, der die für Propagandazwecke konstruierte, mythische Vision der besetzten deutschen Städte begründen soll: 
Ansehen und welterneben!
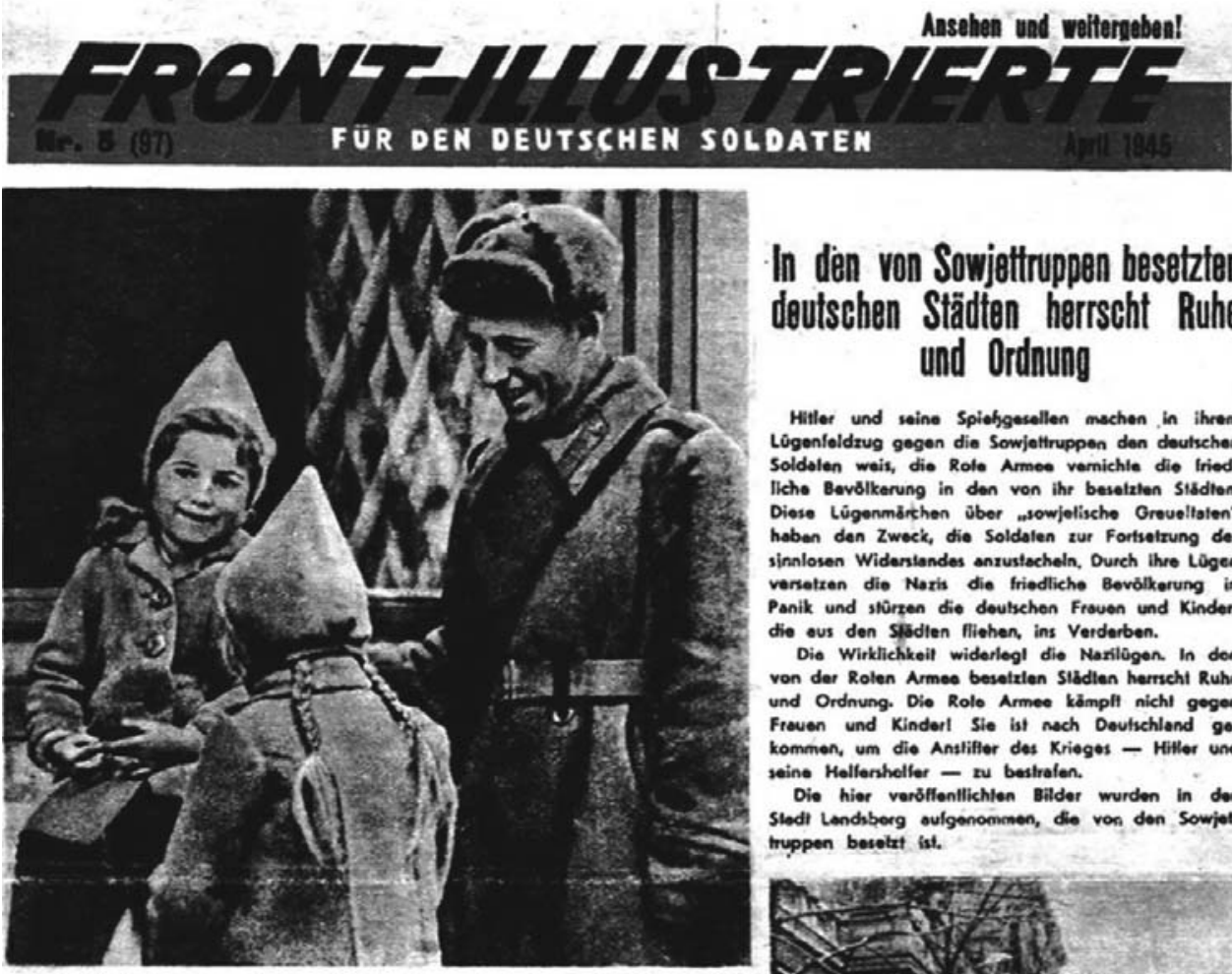

Oleich in den ersten Tagen nech dem Einmench der Roten Armee in Landr

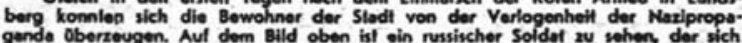
gende oberzeugen. Aut dom Bild oben ist

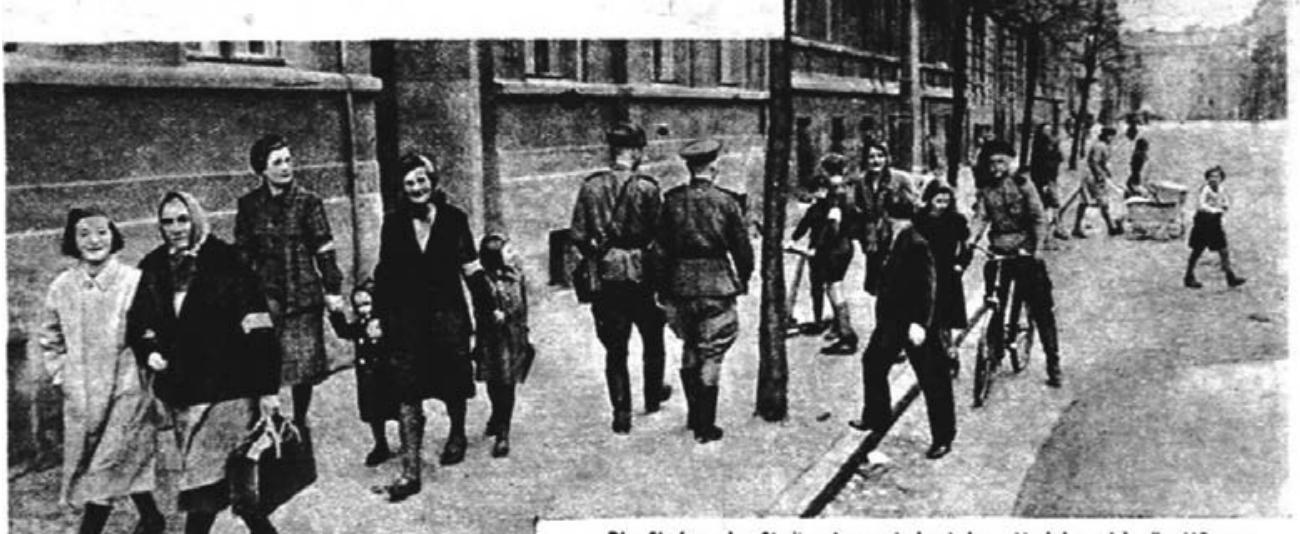

In den von Sowjettruppen besetzten deutschen Städten herrscht Ruhe und Ordnung

Hitler und seine Spiehgesellen mechen in threm Lögenfoldrug gegen die Sowjettruppen den deutschen Soldalen wais, dio Rolo Armee vernichle die Iried. liche Boydlkerving in den von the besetuten sisditon

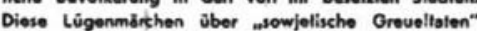
haben den Zwock, dio Soldalen zur Fortsoltrung des sinnlosen Widersiandes anzusischeln, Durch thre Lögen versetzen die Noxis die Friedlicho Bevalikerung in Panik und stürzen die deutsehen Fraven und Kinder, dio ous den stadten fliohen, ins Verdorben.

Die Wirklichkeil widerlegl dio Narilügen. In don von der Rolen Armee besetzlen Siadlen herricht Ruhe und Ordnung. Die Role Armee kämpft nicht gegen Freven und Kindert Sie ist nech Deutuchland gekommen, um die Anstifler des Krieges - Hitlor und seine Hellersheller - zu bestralen.

Dis hier versflentlichten aildar wurden in der

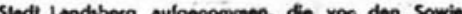
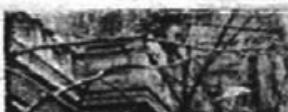

is:

Die Sirakgen dor Slodt zoigon wiedor Laben. Nachdem sich die Männer. Fraven und Kinder Uberzeugl haben, dabs ihnen nichis goschioht, hoben

Abb. 2. Quelle: Hasse-Dresing (Hg.) (2005: 99) 
„Die Wirklichkeit widerlegt die Nazilügen. In den von der Roten Armee besetzten Städten herrscht Ruhe und Ordnung. Die Rote Armee kämpft nicht gegen Frauen und Kinder! Sie ist nach Deutschland gekommen, um die Anstifter des Krieges - Hitler und seine Helfershelfer - zu bestrafen."

Unmittelbar an den letzten Absatz im ersten Teil des Bildberichts schließt sich eine realitätsferne Propaganda-Erzählung über die Zustände in der Stadt an - eine Erzählung, die die vorausgehenden Behauptungen über die Lebensverhältnisse der Deutschen in den besetzten Gebieten beweisen soll: „Die hier veröffentlichten Bilder wurden in der Stadt Landsberg aufgenommen, die von den Sowjettruppen besetzt ist." Im Bildmaterial wird Landsberg zu einer symbolischen Stadt umfunktioniert, die den von der Roten Armee verkündeten Frieden verkörpert. Landsberg soll repräsentativ für alle von den Sowjets besetzten Orte stehen. Unter dem ersten Bild ist folgende Behauptung zu lesen: „Gleich in den ersten Tagen nach dem Einmarsch der Roten Armee in Landsberg konnten sich die Bewohner der Stadt von der Verlogenheit der Nazipropaganda überzeugen." Dies soll das Foto eines russischen Soldaten illustrieren, „der sich freundlich mit deutschen Kindern unterhält.“

Auf dem zweiten Bild sieht man die Deutschen mit den für die damalige Zeit charakteristischen weißen Armbinden als Zeichen dafür, dass auf Widerstand verzichtet wurde. Der Kommentar unter der - womöglich inszenierten - Fotografie schafft das Bild einer deutsch-sowjetischen „post-landsbergischen“ Welt: einer Wirklichkeit, die - so die Absicht der Sieger - im Gedächtnis nicht nur der deutschen Soldaten, sondern vermutlich der ganzen deutschen Bevölkerung zurückbleiben soll. Mehr noch: Man kann die Behauptung wagen, dass die ungewöhnliche Leichtigkeit, mit der das „idyllische“ Propaganda-Bild von Landsberg und seinen Bewohnern nach der russischen Besetzung kreiert wurde, auf das Bedürfnis zurückging, die Sieger (zu denen ja auch die Mitglieder des sowjetischen Propaganda-Apparats gehörten) als Helden erscheinen zu lassen - als edle, ehrenhafte Menschen, die es verdient haben, als Verteidiger des Friedens bezeichnet zu werden.

Für den hier angeführten Propaganda-Text kann eine besondere Gattungsbezeichnung geprägt werden: ,Fantasie-Reportage von der Front'. In diesem Begriff enthalten ist sowohl eine vom Wunschdenken dominierte Weltsicht, als auch eine anonyme Autorschaft (Reporter). An dem Bericht aus der „Front Illustrierten“ fällt das einwandfreie Deutsch auf, das eine Sorge um jedes Detail der Erzählung verrät und womöglich auf die Nationalität des Übersetzers hinweist (vgl. TABORSKa 2017: 71-74).

Um den Grad der Unwirklichkeit des sowjetischen Narrativs vom ,Grenzgebiet des Augenblicks' zu veranschaulichen, gehe ich nun auf Auszüge aus den Memoiren von Florian Kroenke ein - dem ersten polnischen Starosten von Gorzów Wielkopolski, der im März 1945 zusammen mit einer Gruppe von weiteren Polen in der Stadt ankam. In seiner Erzählung erwähnt Kroenke ein (heute nicht mehr erhaltenes) materielles Zeugnis aus dem ,Grenzgebiet des Augenblicks', nämlich zweisprachige (deutsche und polnische) Plakate, die zur Bekämpfung der epidemisch auftretenden Geschlechtskrankheiten aufriefen. Infolge der von Rotarmisten verübten massenhaften Vergewaltigungen breiteten sich die Infektionen schnell aus. Eine umfangreiche Recherche nach diesen Plakaten hat Zofia Nowakowska, Mitarbeiterin des Lebuser Jan-Dekert-Museums und Begründerin des Pionierklubs in Gorzów Wielkopolski, vorgenommen. Von diesen Plakaten hat Nowakowska durch die ersten polnischen 
Stadtbewohner erfahren. Bemerkenswerterweise durfte Florian Kroenke in seinen 1987 publizierten Erinnerungen weder die Ursachen der in Gorzów grassierenden Krankheiten noch sonstige Gräueltaten der Rotarmisten erwähnen:

\begin{abstract}
„Die administrativen Tätigkeiten in Gorzów begannen mit der Einleitung von Hilfsmaßnahmen [für die Deutschen - K.T.]. In der Stadt wurde auf Plakaten eine Anordnung des polnischen Starosten verbreitet, aus der hervorging, dass der Kreisarzt gynäkologische Untersuchungen organisiert hat und dass eine allgemeine Aktion unentgeltlicher Behandlung von Geschlechtskrankheiten eingeleitet wurde. Es war keine rein symbolische Hilfeleistung, sondern eine groß angelegte Aktion. Mehr als ein Dutzend Ärzte sowie das Hilfspersonal waren daran beteiligt. Zugleich wurde in Gorzów und der Umgebung eine Sammlung von Medikamenten angeordnet und die Produktion medizinischer Kohle in Gang gesetzt - nicht nur für die Zivilbevölkerung, sondern auch für das Militär. Wir haben diese Maßnahmen ergriffen, als wir die Meldung bekamen, dass in der Stadt Geschlechtskrankheiten und Ruhr um sich greifen. Da das Leben völlig durcheinander geraten war und die Versorgung mit Medikamenten vollkommen versagte, erfüllte dieser Missstand die Zivilbevölkerung mit tiefer Besorgnis und verstärkte die allgemein herrschende Atmosphäre der Verzweiflung. Man erwartete verschiedene Komplikationen und gefährliche Konsequenzen (es seien bereits mehrere Fälle von Bauchtyphus festgestellt worden). Die Maßnahmen, die wir einleiteten, vermittelten eine eindeutige Botschaft und haben die Atmosphäre von Beunruhigung und Ratlosigkeit entspannt.“" (KROENKe 1987: 74)
\end{abstract}

Es ist unschwer zu erkennen, dass die beiden hier besprochenen Texte: Kroenkes Bericht aus dem Band Wiosna na rumowisku (,Frühling auf dem Trümmerhaufen') und die ,Fantasie-Reportage von der Front' lediglich ein einziger Fakt verbindet: Beide schildern dieselbe Stadt zu der Zeit, in der dort sowjetische Truppen stationiert waren (vgl. TABORSKA 2017: 74).

3.

Der im vorigen Abschnitt erwähnte Aufruf des Bürgermeisters Paul Schultz verdient besondere Beachtung. Er enthält einen Satz (im nachfolgenden Zitat durch Sperrdruck hervorgehoben), den polnische Historiker übernommen haben, der aber keineswegs den Fakten entspricht. Unten zitiere ich den vollständigen Text des Aufrufs (ausgelassen werden lediglich die Unterschriften der Verwaltungsmitarbeiter), um aufzuzeigen, mit welchen Methoden die sowjetische Militärkommandantur versuchte, die Landsberger, die der dramatischen Lage verzweifelt und hoffnungslos gegenüberstanden, zu beruhigen und zu disziplinieren. Zur Kommunikation mit der Stadtbevölkerung bedienten sich die Besatzer einer selbst ernannten Verwaltung mit dem Bürgermeister an der Spitze. Wohlgemerkt wird die Westverschiebung Polens im Text nicht erwähnt.

„Aufruf des von der Besatzungsmacht eingesetzten Bürgermeisters Paul Schultz

Deutsche!

Wir, die Einwohner der Stadt Landsberg (Warthe), wenden uns an Euch jenseits der Front, um Euch den Weg zur Rettung zu zeigen. Jetzt, nachdem die Kämpfe durch unsere Stadt gerollt sind, können wir offen und ehrlich, ohne Angst vor der Gestapo, die Wahrheit sagen, wie die Naziführung Euch im Unklaren läßt. Wir haben unser Leben und das Leben unserer Angehörigen darum bewahrt, weil wir begriffen haben, daß Hitlers Krieg verloren und weiterer Widerstand gegen die Rote Armee sinnlos ist. Darum haben wir unsere Stadt mit ihren Häusern, Kirchen, Kulturstätten, Fabriken und Werken 
bewahrt, weil wir begriffen haben, daß wir die unausbleibliche Vernichtung der Hitlerarmee nicht verhüten können. Darum haben wir unser Hab und Gut bewahrt, weil wir es nicht zum Opfer bringen wollten, um nur kurze Zeit den unausbleiblichen Zusammenbruch des Naziregimes zu verhindern. Darum sind wir dem Schrecken der Evakuierung entgangen, weil wir der Nazipropaganda keinen Glauben betr. der Greultaten der Bolschewisten geschenkt haben. Wir haben uns nicht getäuscht und glauben an die Beschlüsse der Krimkonferenz, in denen es u.a. heißt:

,Zu unseren Zielen gehört nicht die Vernichtung des deutschen Volkes. Erst wenn der Nazismus und Militarismus ausgerottet sind, besteht für das deutsche Volk die Hoffnung auf eine würdige Existenz und einen Platz in der Gemeinschaft der Nationen.

Für uns ist der Krieg beendet. Unser Leben ist in Sicherheit. Der russische Militärkommandant gewährleistet die Ordnung in der Stadt. Unser Leben verläuft normal. In unserer Stadt ist zivile Verwaltung schon gebildet. Der Bürgermeister ist Paul Schultz. Unter seiner Leitung arbeiten die Abteilungen:

1) Versorgungsabteilung (Lebensmittel).

2) Abteilung zur Entgegennahmen von Beschwerden der zivilen Bevölkerung.

3) Abteilung Sanitätswesen.

4) Hilfe für Obdachlose, zurückgekommene Flüchtlinge, Kinder und Invaliden.

5) Abteilung für Industrie und Handel.

Das städtische Elektrizitätswerk und Wasserwerk funktionieren störungsfrei. 2 Krankenhäuser und 9 zurückgebliebene Ärzte arbeiten für die zivile Bevölkerung. 14 Bäckereien versorgen die Zivilbevölkerung mit Brot. Jeder Arbeitnehmer bekommt 400g Brot pro Tag, die nicht arbeitende Bevölkerung erhält $200 \mathrm{~g}$ Brot pro Tag. In vollem Betrieb ist eine Fleischwarenfabrik. In kürzester Frist werden auch die anderen Betriebe der Stadt in Gang kommen. Die Kirchen stehen wie zuvor. Gottesdienste können abgehalten werden. Deutsche, Hitler hat den Krieg verloren. Hitler will um den Preis Eures Lebens seine Existenz hinauszögern, laßt nicht zwecklos Euer Leben und das Leben Eurer Angehörigen zugrunderichten. Helft nicht den Naziführern, Eure Heimstätte zum Kampfplatz zu verwandeln. Ihr müßt begreifen, dass die Schützengräben und alle anderen Befestigungen, die Ihr baut, Eure Gräber sein werden. Wir haben selbst monatelang diese Schützengräben und Befestigungen gebaut, aber alles war vergebens. Die Keller Eurer Häuser, die Ihr zu Befestigungen macht, werden Euch selbst unter Schutt begraben. Der Eintritt in den Volkssturm bedeutet Selbstmord. Wer dem Aufruf zur Evakuierung Folge leistet, geht der Armut und dem Hungertode entgegen. Deutsche, laßt Euch unser Beispiel als Weg zur Rettung dienen. Euer Leben ist in Eurer Hand. Brecht mit Hitler und seinem Regime! Verweigert alle Anordnungen und Befehle der Naziführer. Laßt Eure Söhne nicht in den Volkssturm und tretet selber nicht ein. Überzeugt alle Angehörigen der Wehrmacht, daß je schneller sie kapitulieren, desto schneller wird dieses Unglück des deutschen Volkes zu Ende sein. Ihr müßt begreifen, wie wir es begriffen haben, daß das deutsche Volk nur bei sofortiger Kapitulation seine Stellung in der Gemeinschaft der Nationen wieder einnehmen kann. Glaubt nicht, daß wir Euch zum Verrat aufrufen wollen, sondern nur zur Rettung der Heimat. Das Schicksal Hitlers und seiner Anhänger ist besiegelt. Vergeßt nicht, Hindenburg und Ludendorf haben 1918 klugerweise den Krieg beendet, als er für Deutschland verloren war. Der Gefreite Hitler begreift den Ernst der Lage nicht. Das Naziregime in Deutschland muß ausgerottet werden, das deutsche Volk aber muß leben. Die Weiterexistenz des Hitlerregimes bringt dem deutschen Volk neue Entbehrungen, Strapazen und weitere Verwüstungen Deutschlands. Je schneller Ihr Hitler und seinen Anhängern den Rücken kehrt, desto schneller haben Eure Not, Euer Elend und alle Entbehrungen ein Ende. Handelt, ehe es zu spät ist. Deutsches Volk, nun handle selbst! [...]"

(HASSE-Dresing (Hg.) 2005: 100-101, Schreibweise wie im Original) 
In dem durch Sperrdruck hervorgehobenen Satz ist von Kämpfen die Rede, die durch die Stadt „gerollt" seien. Wiederholen wir es aber noch einmal: Landsberg wurde ohne jeglichen Widerstand besetzt. Vor dem Einmarsch der Rotarmisten haben sich die deutschen Truppen aus der Stadt zurückgezogen. Die enormen Verwüstungen, die Sowjet-Soldaten angerichtet haben, werden in dem Aufruf indirekt durch die angeblich in der Stadt ausgetragenen Kämpfe begründet. Dasselbe Motiv, das im angeführten ephemeren Druck verwendet wird, ist auch bei polnischen Historikern anzutreffen. So zählt RYmaR (2005: 22-23) diejenigen Autoren auf, die von den „Kämpfen um Landsberg" sprechen, und weist darauf hin, dass diese Version der Ereignisse in der polnischen Geschichtsschreibung jahrzehntelang gültig war. Das Motiv, sowohl in den deutschsprachigen als auch in den polnischen Varianten eine mythische Geschichte zu kreieren, war dasselbe: Man wollte eine plausible Erklärung für die Vernichtung der Stadt liefern. Paradoxerweise stützen sich sämtliche polnischen Erzählungen von den angeblichen Kämpfen um Landsberg auf eine einzige Quelle: eine Behauptung in deutscher Sprache, die nach Anweisungen der sowjetischen Militärkommandantur verfasst worden war.

Die wenigen hier dargestellten Beispiele für Ephemera, die im ,Grenzgebiet des Augenblicks' entstanden, veranschaulichen die außergewöhnliche Resistenz von derartigen Texten bzw. Artefakten sowie ihre Kraft, das Bild der Realität mitzugestalten. Tatsächlich handelt es sich dabei um einige der ersten Schriftstücke, die die gesellschaftlichen Vorstellungen von den ,neuen Grenzgebieten' mitgeprägt haben. Dieses prä-narrative Potential macht Ephemera zu einem wertvollen Forschungsgegenstand, der sich aus vielfältigen kulturellen und transdisziplinären Perspektiven betrachten lässt.

Aus dem Polnischen von Katarzyna Lukas

\section{Bibliographie}

Adamczews Ki, Leszek (2012): Berlińskie wrota. Nowa Marchia w ogniu [Das Berliner Tor. Die Neumark im Feuer]. Zakrzewo: Wydawnictwo Replika.

[Anonym] (1962): „Tötet, tötet, tötet!“ (Rezension zu: Ilja Ehrenburg, Menschen, Jahre, Leben, München 1962: Kindler). In: „Der Spiegel“ 36/1962 (05.09.1962), 71-76 (https://www.spiegel.de/ spiegel/print/d-45141455.html, 15.06.2019).

Hasse-Dresing, Ursula (Hg.) (2005): Quellen und Darstellungen zur Geschichte des Landsberger Landes (3). Herford: Eigenverlag von der Bundesarbeitsgemeinschaft Landsberg (Warthe) Stadt und Land e.V.

Kroenke, Florian (1987): Zamiast wstępu [Anstatt einer Einführung]. In: Linkowski, Zdzisław (Hg.): Wiosna na rumowisku i inne wspomnienia pionierów [Der Frühling auf dem Trümmerhaufen und andere Erinnerungen der Pioniere]. Gorzów Wielkopolski: Gorzowskie Towarzystwo Kultury, 5-23.

Meyer-Ort, Hartmut (2008): Gorzow / Landsberg in meinem Gedächtnis 1926 - August 1945. In: Jaworski, Edward / Kost KIEwiCz-Górska, Grażyna / Skawiński, Błażej (Hg.): Gorzów 
w mojejpamięci / Gorzow in meiner Erinnerung. Übers. v. Kowalski, Grzegorz. Gorzów Wielkopolski: Wojewódzka i Miejska Biblioteka Publiczna w Gorzowie Wielkopolskim, 99-230.

TABORSKa, Katarzyna (2017): Literatura miejsca. Piśmiennictwo postlandsberskie [Literatur des Ortes. Das post-landsbergische Schriftum]. Gorzów Wielkopolski: Wydawnictwo Naukowe Akademii im. Jakuba z Paradyża.

Rymar, Dariusz Aleksander (2005): Gorzów Wielkopolski w latach 1945-1998. Przemiany spoteczno-polityczne [Gorzów Wielkopolski 1945-1998. Sozial-politischer Wandel]. Szczecin - Gorzów Wielkopolski: Wydawnictwo „Dokument”.

RymaR, Dariusz Aleksander (Hg.) (2001): Trudne gorzowskie początki (Teksty źródtowe z lat 1945-1948) [Die schwierigen Anfänge von Gorzów Wielkopolski (Quellentexte aus den Jahren 1945-1948)]. Gorzów Wielkopolski: Towarzystwo Przyjaciół Archiwum i Pamiątek Przeszłości.

Rymar, Dariusz Aleksander (2015): Gorzów - stolica Ziemi Lubuskiej w latach 1945-1950 [Gorzów - die Hauptstadt vom Land Lebus 1945-1950]. Gorzów Wielkopolski: Archiwum Państwowe w Gorzowie Wielkopolskim.

RżEwska, Elena (1975): Berlin, maj 1945 [Berlin, Mai 1945]. Aus dem Russischen von Bogdański, Aleksander. Warszawa: Czytelnik.

РЖевСКАЯ, Елена: Берлин, май 1945, http://militera.lib.ru/memo/russian/pzhevskaya_em2/index. html (15.06.2019).

Venclova, Tomas (2003): Kwadratura koła [Die Quadratur des Kreises], http://wyborcza. $\mathrm{pl} / 1,75410,1299216 . \mathrm{html}(15.06 .2019)$.

ZARZYCKA-BÉRARD, Ewa (2002): Burzliwe życie Ilii Erenburga. Rosyjski los, żydowskie szczęście [Das bewegte Leben des Ilja Ehrenburg. Russisches Schicksal, jüdisches Glück]. Aus dem Französischen von KozaK, Agata. Warszawa: Iskry. 